\title{
Baryogenesis via gravitational spontaneous symmetry breaking
}

\author{
Qiuyue๑, ${ }^{*}$ Jeremy Sakstein, ${ }^{\dagger}$ and Mark Trodden \\ Center for Particle Cosmology, Department of Physics and Astronomy, University of Pennsylvania, \\ 209 South 33rd Street, Philadelphia, Pennsylvania 19104, USA
}

(Received 19 June 2019; published 17 September 2019)

\begin{abstract}
We study baryogenesis in effective field theories where a $\mathrm{U}(1)_{B-L}$-charged scalar couples to gravity via curvature invariants. We analyze the general possibilities in such models, noting the relationships between some of them and existing models, such as Affleck-Dine baryogenesis. We then identify a novel mechanism in which $\mathrm{U}(1)_{B-L}$ can be broken by couplings to the Gauss-Bonnet invariant during inflation and reheating. Using analytic methods, we demonstrate that this mechanism provides a new way to dynamically generate the net matter-antimatter asymmetry observed today and verify this numerically.
\end{abstract}

DOI: 10.1103/PhysRevD.100.063518

\section{INTRODUCTION}

There is more matter than antimatter in the Universe. This fact by itself is hardly surprising: The baryon and lepton number are accidental symmetries of the standard model that are broken by higher-dimensional operators and nonperturbative objects (sphalerons) [1-10] so that the only conserved quantity is their difference $B-L$. One would therefore expect some asymmetry in the Universe, the exact amount of which can be quantified by the baryon to entropy ratio $n_{\mathrm{B}} / n_{\mathrm{s}}$, a quantity that is observed to be $\sim 10^{-10}$ $[11,12]$. Whether or not the standard model can account for this depends on the magnitude of $C P$-violating effects and, crucially, on the nature of the electroweak phase transition. In particular, only a first-order transition can produce a sufficient asymmetry. The measurement of the Higgs mass at $125 \mathrm{GeV}$ (e.g., [13]) implies that the transition is a crossover, and therefore some new physics beyond the standard model (BSM) is required to generate the observed asymmetry dynamically.

There is a profusion of BSM mechanisms and models that could generate such an asymmetry (see [3,14-16] for reviews). Many BSM theories, including supersymmetric theories, include new scalar particles that are charged under $\mathrm{U}(1)_{B-L}$ (this is a consequence of the fact that this combination is anomaly-free), and several baryogenesis mechanisms employ a breaking of $B-L$ to achieve a

\footnotetext{
*qyliang@sas.upenn.edu

sakstein@physics.upenn.edu

trodden@physics.upenn.edu
}

Published by the American Physical Society under the terms of the Creative Commons Attribution 4.0 International license. Further distribution of this work must maintain attribution to the author(s) and the published article's title, journal citation, and DOI. Funded by SCOAP. dynamical generation of the asymmetry, transferring it to the standard model at a later time or through sphaleron processes. A particularly well-studied mechanism for breaking the symmetry in these scenarios is the AffleckDine mechanism $[4,7,17,18]$. Here, the $\mathrm{U}(1)_{B-L}$ can be broken during single-field slow-roll inflation or reheating due to a coupling between the scalar and the kinetic and potential energy and the inflaton. If the reheating is perturbative-i.e., the inflaton is oscillating about the minimum of its potential - then this coupling will dominate over any nonderivative couplings, and baryogenesis may proceed due to the symmetry breaking. However, the process of reheating is likely more complicated and nonlinear than simple perturbative scenarios $[19,20]$. Similarly, inflation may be more complicated than single-field slow roll. This has motivated recent searches for symmetrybreaking mechanisms that are less sensitive to the precise details of inflation and reheating or decoupled from them completely. In particular, Ref. [21] presented a new model where a coupling of a scalar to dark matter or, equivalently, to the Ricci scalar (see also [22,23]) can provide the symmetry breaking needed without any reference to inflation whatsoever. Similarly, Ref. [24] constructed a similar mechanism in which the symmetry is instead broken by a coupling to a new vector that spontaneously breaks Lorentz invariance when it aligns with the cosmic rest frame.

The purpose of this paper is to systematically study the different mechanisms by which a U(1) symmetry can be broken by gravitational effects. To accomplish this, we consider the low-energy effective field theory (EFT) of a $\mathrm{U}(1)$-charged scalar (that we will eventually identify with $B-L$ ) coupled to gravity via curvature tensors. We will also include potential couplings to the inflaton for illustrative purposes and to make contact with the Affleck-Dine literature. In doing so, we will identify a new symmetrybreaking mechanism that arises due to a coupling between 
the scalar and the Gauss-Bonnet (GB) invariant. This symmetry breaking exhibits novel features compared with the Affleck-Dine and scalar-dark matter scenarios (as well as other scalar-Gauss-Bonnet [25] baryogenesis scenarios), which we exploit to devise a new mechanism for baryogenesis: During inflation, the symmetry is broken and the field sits at its constant, symmetry-breaking minimum. After inflation, and during reheating, the GB term redshifts, which causes the symmetry-breaking minimum to move closer to zero, and the symmetry is ultimately restored once this term has redshifted sufficiently. During this time, the motion of the angular field generates a net $B-L$ dynamically. After reheating, the symmetry is restored so that the field has a zero vacuum expectation value (VEV). The asymmetry is later transferred to the standard model via couplings of the scalar to the left-handed sector, for example, via a neutrino portal. This coupling therefore allows for a baryogenesis scenario with some similarities to the Affleck-Dine mechanism but for more general reheating (and preheating) mechanisms (e.g., [26]). It is often the case that models where scalars couple to the Ricci scalar do so in a manner that does not give rise to spontaneous symmetry breaking [27-30]. In these cases, baryogenesis cannot occur unless the symmetry is broken by other terms in the EFT after inflation. Our mechanism is the first example of this.

Scalar fields coupled to the Gauss-Bonnet invariant are ubiquitous in cosmology and gravitational physics, and they also arise in the low-energy effective action of certain string compactifications [31]. In cosmology, they may play the role of dark energy [32-34] and can drive inflation [35-38]. They are one of the few theories that are known to violate the nohair theorem for black holes [39], which has made them the focus of current strong gravity research. Theoretically, these couplings are of interest, since they do not introduce new ghost degrees of freedom, so that the resultant theories are healthy (provided one does not choose phantom kinetic terms or a potential that is unbounded from below). Despite the intense theoretical interest in these models, to our knowledge, there have been no investigations into whether these theories include a mechanism for baryogenesis and whether they have the potential to produce the matterantimatter asymmetry we observe in our Universe. We will demonstrate in this paper that, indeed, they can.

The paper is organized as follows: In the next section, we take an effective field theory approach to categorizing how gravitational effects can give rise to baryogenesis in the types of models discussed above. In Sec. III, we then focus on a novel baryogenesis mechanism employing a coupling to the Gauss-Bonnet invariant and study its implications before summarizing our results in the final section.

\section{BARYOGENESIS WITH SCALAR-GRAVITY COUPLINGS}

The building blocks of our theory are a massless spin-2 graviton $g_{\mu \nu}$ and a new scalar $\phi$ which has charge $q$ under
$\mathrm{U}(1)_{B-L}$. In order to make contact with the Affleck-Dine mechanism, we will also include an inflaton field $\Phi$, but this will play no role in the mechanism described in this work. The action is

$$
\begin{aligned}
S= & \int \mathrm{d}^{4} x \sqrt{-g}\left[\frac{M_{\mathrm{pl}}^{2}}{2} R-\frac{1}{2} \nabla_{\mu} \Phi \nabla^{\mu} \Phi-\nabla_{\mu} \phi \nabla^{\mu} \phi^{\star}\right. \\
& -m_{\phi}^{2}|\phi|^{2}-\lambda|\phi|^{4}-c \frac{|\phi|^{2}}{\mathcal{M}^{2}} \nabla_{\mu} \Phi \nabla^{\mu} \Phi-V_{\mathrm{inf}}(\Phi) \\
& \left.-V_{\mathrm{int}}(\Phi,|\phi|)-\alpha|\phi|^{2} R \pm \frac{|\phi|^{2}}{\mathcal{M}^{2}} \mathcal{G}\right],
\end{aligned}
$$

where $\mathcal{G}=R^{\alpha \beta \mu \nu} R_{\alpha \beta \mu \nu}-4 R_{\mu \nu} R^{\mu \nu}+R^{2}$ is the Gauss-Bonnet invariant. Some explanation is in order. Equation (1) is the effective field theory for our new scalar coupled to gravity and the inflaton up to dimension-six operators, with the cutoff denoted by $\mathcal{M}$. We have not included every possible coupling (e.g., inflaton-Gauss-Bonnet, $G^{\mu \nu} \partial_{\mu} \phi \partial_{\nu} \phi$, or $\Phi^{2} R$, for example) in the interest of compactness; these operators are either unimportant or do not qualitatively change the dynamics. The inflaton self-potential $V_{\text {inf }}(\Phi)$ is responsible for driving inflation, while the interaction potential $V_{\text {int }}(\Phi,|\phi|)$ represents other possible interactions with the scalar $\phi$ that are not forbidden by symmetries. They are included for completeness and play no role in what follows, so we will not concern ourselves with their specific form. Finally, we must supplement Eq. (1) with symmetry-violating terms

$$
S_{\text {symmetry violating }}=-\int \mathrm{d}^{4} x \sqrt{-g}\left[\frac{\varepsilon}{4} \phi^{4}+\frac{\varepsilon^{\star}}{4} \phi^{\star 4}\right] .
$$

These operators are ubiquitous in any theory where there are several new scalar degrees of freedom [18]. For example, if the $\mathrm{U}(1)_{B-L}$ charge is $q$ and there is another heavy charged field $\chi$ with charge $4 q$, the leading-order interaction is

$$
S_{\phi \chi}=-\int \mathrm{d}^{4} x \sqrt{-g}\left[\frac{\phi^{4} \chi^{*}}{\overline{\mathcal{M}}}+\text { H.c. }\right] .
$$

If $\chi$ acquires a nonzero $\mathrm{VEV}$, then we can integrate it out to find precisely Eq. (2) with

$$
\varepsilon=4 \frac{\left\langle\chi^{\star}\right\rangle}{\overline{\mathcal{M}}} .
$$

In this example, we expect $\left\langle\chi^{\star}\right\rangle \ll \overline{\mathcal{M}}$ so that $|\varepsilon| \ll 1$.

Equations (1) and (2) imply an effective potential for $\phi$ :

$$
\begin{aligned}
V_{\text {eff }}\left(\phi, \phi^{\star}\right)= & \left(m_{\phi}^{2}+c \frac{\dot{\Phi}^{2}}{\mathcal{M}^{2}}+\alpha R \pm \frac{\mathcal{G}}{\mathcal{M}^{2}}+V_{\text {int }, \phi \phi^{*}}(\bar{\Phi})\right)|\phi|^{2} \\
& +\lambda|\phi|^{4}+\varepsilon \frac{\phi^{4}}{4}+\varepsilon^{\star} \frac{\phi^{\star 4}}{4}
\end{aligned}
$$


where $\dot{\Phi}^{2}$ is the kinetic energy of the inflaton and $\bar{\Phi}$ is the background value of the inflaton. We will see presently that the sign of the Gauss-Bonnet term depends on the equation of state, so we have included both signs for now in order to be general. Note also that the effective potential can either be symmetry preserving or symmetry breaking, depending on the sign of the coefficient of the quadratic term. In particular, if this term is negative, the symmetry can be broken, which allows for the possibility of baryogenesis. By inspection, one can discern four possibilities for this. First, if $V_{\mathrm{int}, \phi \phi^{*}}(\bar{\Phi})<0$, then the scalar's mass is tachyonic during inflation. Second, if $c<0$, then the kinetic coupling of $\phi$ to the inflaton can drive a symmetry breaking. The Affleck-Dine mechanism $[4,17,18]$ utilizes a combination of these two possibilities. In this case, one has $\dot{\Phi}^{2} \sim$ $H^{2} M_{\mathrm{pl}}{ }^{2} / \mathcal{M}^{2}$ so that the symmetry-breaking term is proportional to $c\left(M_{\mathrm{pl}}^{2} / \mathcal{M}^{2}\right) H^{2}$, and, furthermore, the cosmological dynamics of the inflaton are such that are $V_{\mathrm{int}, \Phi \Phi}(\bar{\phi}) \propto-c^{\prime} H^{2}$ for some $c^{\prime}>0$ and $\mathcal{O}(1)$ [18]. Third, if $\alpha<0$ and there is some amount of nonrelativistic matter present, then the symmetry can be broken, because $R \sim \rho_{\mathrm{m}} / M_{\mathrm{pl}}{ }^{2}$. This is the mechanism of Refs. [21,22]. Finally, if $\pm \mathcal{G}<0$ - with the sign chosen depending on the sign of $\mathcal{G}$ - then the symmetry can be broken by the GaussBonnet invariant. This possibility has not been considered previously, and so we will focus on this exclusively in the present work. ${ }^{1}$

For a Friedmann-Robertson-Walker universe

$$
\mathrm{d} s^{2}=-\mathrm{d} t^{2}+a^{2}(t)\left(\mathrm{d} r^{2}+r^{2} \mathrm{~d} \Omega_{S^{2}}^{2}\right)
$$

dominated by a perfect fluid with equation of state $w$, the Gauss-Bonnet invariant is

$$
\mathcal{G}= \begin{cases}-\frac{64(1+3 w)}{27(1+w)^{4} t^{4}} & w \neq-1, \\ 24 H^{4} & w=-1\end{cases}
$$

This implies that, if we choose the negative sign in Eq. (5), the symmetry is broken when $-1 \leq w<-1 / 3$ (i.e., whenever the universe is accelerating, including during an exact de Sitter phase). If, instead, we choose the positive sign, then the symmetry is broken if $w>-1 / 3$ (i.e., whenever the universe is decelerating). Therefore, the choice of sign is determined by the cosmological epoch during which one requires the symmetry to be broken. In order to decide this, it is instructive to consider the symmetry-breaking minimum, which, using Eq. (5), is given by (ignoring the Riccicoupling and the symmetry-breaking terms for now)

\footnotetext{
${ }^{1}$ Note that the bounds on scalar-gravity couplings of this form from GW170817 [40-43] do not apply to our models, since the scalar is cosmologically subdominant throughout the entire Universe's history [44].
}

$$
|\phi|_{\min }=\frac{1}{\sqrt{2 \lambda}}\left(\left|\frac{\mathcal{G}}{\mathcal{M}^{2}}\right|-m_{\phi}^{2}\right)^{1 / 2} .
$$

During inflation (or, to be more precise, in exact de Sitter space), the position of this minimum is constant, but on a general cosmological solution it is time dependent, and one has

$$
|\phi|_{\min }=\frac{1}{\sqrt{2 \lambda}}\left(\frac{64|(1+3 w)|}{27(1+w)^{4} \mathcal{M}^{2} t^{4}}-m_{\phi}^{2}\right)^{1 / 2} .
$$

This is the first difference between Gauss-Bonnet-induced symmetry breaking and Affleck-Dine or dark-matterinduced baryogenesis. In the latter two mechanisms, the symmetry-breaking term redshifts as $t^{-2}$, whereas in the former it redshifts as $t^{-4}$, allowing for a novel phenomenology. Considering an epoch where $w$ can be treated as a constant, the symmetry is restored at a time

$$
\bar{t}^{4}=\frac{64|(1+3 w)|}{27(1+w)^{4} \mathcal{M}^{2} m_{\phi}^{2}} .
$$

Now, since there is a strong time dependence, it is not necessarily the case that the minimum is an attractor. Indeed, changing variables to

$$
t=\bar{t} e^{z}, \quad \xi=\frac{\phi(z)}{\phi_{\min }(z)},
$$

the equation of motion for the scalar at early times when $t \ll \bar{t}$ is

$\xi^{\prime \prime}-\frac{3+5 w}{1+w} \xi^{\prime}+2 \frac{1+3 w}{1+w} \xi+m_{\phi}^{2} \bar{t}^{2} e^{-2 z} \xi\left(\xi^{2}-1\right)=0$.

The equivalent equation for Affleck-Dine or scalar-dark matter mechanisms has a fixed point near $\xi=1$ (the fixed point is not exactly at $\xi=1$ due to nonlinear effects and cosmological time dependence) [18]. In our case, setting $\xi^{\prime}=\xi^{\prime \prime}=0$, one has

$$
\xi^{2}=1-2 \frac{e^{2 z}}{m_{\phi}^{2} \bar{t}^{2}} \frac{1+3 w}{1+w}
$$

so that on long enough timescales (i.e., $t \gg \bar{t}$ ) the field will move away from the minimum exponentially. This is a new feature of the scalar-Gauss-Bonnet coupling. In practice, we are interested in the dynamics when $t \ll \bar{t}$-in fact, the approximations used to derive Eq. (11) break down at times $t \sim \bar{t}$ - so this instability is not important. The important term for determining the stability in Eq. (11) is the second one, which represents a negative friction that will drive the field away from the minimum unless $-1 \leq w<-3 / 5$. This means that, in order for baryogenesis to proceed in the absence of fine-tuning, any mechanism based on a 
scalar-Gauss-Bonnet coupling must necessarily occur during an epoch where the equation of state lies within this range. Clearly, this is not the case during the matter- and radiation-dominated eras. The patent epoch is reheating, which occurs at some time between the end of inflation, where the equation of state $w \approx-1$, and the start of the matter era, where $w=1 / 3$. $^{2}$ The scenario we will therefore develop envisions that during reheating the equation of state changes from $w=-1$ to $w=1 / 3$ due to the production of radiation. During this time, the symmetry is broken and the minimum is an attractive fixed point, allowing for stable baryogenesis. We will now derive the dynamics of this mechanism in detail.

\section{BARYOGENESIS FROM A GAUSS-BONNET COUPLING}

Having understood the dynamics of symmetry breaking due to a scalar-Gauss-Bonnet coupling, we are now in a position to describe a scenario for baryogenesis using this mechanism. Since such a scenario can be viable only during reheating and when $-1 \leq w<-3 / 5$, we fix the sign of the scalar-Gauss-Bonnet coupling to be negative in Eq. (5) so that the $\mathrm{U}(1)_{B-L}$ can be broken spontaneously during this epoch [see the discussion below Eq. (7)]. The scenario is as follows.

\section{A. Inflation and initial conditions}

During inflation, the absolute value of the field $|\phi|$ sits at the (constant) minimum of the effective potential (5). Considering slow-roll inflation, which is the scenario favored by Planck observations [45], we can ignore the kinetic coupling to the inflaton, and furthermore we neglect the interaction potential in order to study the effects of the Gauss-Bonnet coupling, but one should not ignore the Ricci coupling in a consistent effective field theory. The effective potential for this field is then

$$
V(|\phi|)=\left(m_{\phi}^{2}+12 \alpha H_{I}^{2}-24 \frac{H_{I}^{4}}{\mathcal{M}^{2}}\right)|\phi|^{2}+\lambda|\phi|^{4},
$$

where $H_{I}$ is the Hubble constant during inflation and $R=12 H_{I}^{2}$. Provided that $H_{I}>(\alpha / 2)^{1 / 2} \mathcal{M}$ [one should not take $H_{I} \gg(\alpha / 2)^{1 / 2} \mathcal{M}$, since this would be tantamount to applying the EFT above its cutoff], the symmetry is broken during inflation and the field sits at the minimum, approximately given by

\footnotetext{
${ }^{2}$ One could imagine this process happening during inflation, but this would imply isocurvature modes and could possibly alter the dynamics of inflation itself. It is also highly likely that any net $B-L$ generated would rapidly redshift unless one fine-tunes the parameters to have the requisite amount of $B-L$ produced very close to the end of inflation.
}

$$
\left|\phi_{I}\right| \sim \sqrt{\frac{12}{\lambda}} \frac{H_{I}^{2}}{\mathcal{M}}
$$

The mass at the minimum is of the order of $H_{I}^{2} / \mathcal{M} \gtrsim H_{I}$, so that the field is sufficiently heavy that it does not acquire isocurvature perturbations, nor does it impact the dynamics of the inflaton. Writing $\phi=|\phi| e^{i \theta}$, there is a small potential for the angular field $\theta$ due to the symmetry-breaking terms

$$
\Delta V(\theta)=\frac{\varepsilon_{0}}{2}|\phi|^{4} \cos (4 \theta+\psi),
$$

where we have written $\varepsilon=\varepsilon_{0} \exp (i \psi)$. Recalling that the angular field is not canonically normalized, so that $\mathcal{L} \supset|\phi|^{2}\left(\nabla_{\mu} \theta\right)^{2}$, we infer that the mass for this field is

$m_{\theta}^{2} \sim \varepsilon_{0}\left|\phi_{I}\right|^{2} \cos \left(4 \theta_{0}+\psi\right) \sim \varepsilon_{0} \frac{H_{I}^{2}}{\mathcal{M}} \cos \left(4 \theta_{0}+\psi\right)$,

where $\theta_{0}$ is the initial value of $\theta$ during inflation. Now, since $\varepsilon_{0} \ll 1$, we expect this mass to be sub-Hubble, so that the field is overdamped and is fixed to $\theta_{0}$. In fact, if this is not the case, the field would minimize its potential during inflation and no net $B-L$ would be produced thereafter. This implies that $\theta$ acquires isocurvature perturbations [46-48]

$$
\langle\delta \theta\rangle=\frac{H_{I}}{2 \pi\left|\phi_{I}\right|} .
$$

Since the symmetry-breaking terms are responsible for the generation of the net $B-L$, the amplitude of these perturbations is given by

$$
S_{B \gamma} \equiv \frac{\delta \rho_{B}}{\rho_{B}}-\frac{3}{4} \frac{\delta \rho_{\gamma}}{\rho_{\gamma}}=\frac{\delta n_{B}}{n_{B}},
$$

where the subscript $B$ denotes baryons and the subscript $\gamma \mathrm{s}$ denotes photons. Ultimately, $n_{B}$ arises from $n_{B-L}$ after sphaleron reprocessing of the generated $B-L$. Therefore, we can estimate the amplitude of isocurvature perturbations by estimating $n_{B-L}$. This is generated by the motion of the angular field, so we have

$$
n_{B-L} \propto \partial_{\theta} \Delta V(\theta) \propto \sin (4 \theta+\psi),
$$

which yields $\delta n_{B} / n_{B} \sim 4 \cot \left(4 \theta_{0}+\psi\right) \delta \theta$. Using Eq. (17) and the isocurvature bounds from Planck [45], $S_{B \gamma}<$ $3 \times 10^{-4}$, we have

$$
\frac{H_{I}}{\left|\phi_{I}\right|}=\frac{\pi}{2} \tan \left(4 \theta_{0}+\psi\right) S_{B \gamma} \lesssim 3 \times 10^{-4} .
$$

This imposes a bound on the initial conditions and on the model parameters. We thus restrict to values where this is satisfied. It turns out this is not a very restrictive 
requirement, and we will verify later that it is satisfied by any reasonable model.

\section{B. Reheating and baryogenesis}

When inflation ends, the equation of state increases from -1 through reheating, ultimately ending up at $1 / 3$, which signifies the beginning of the radiation epoch. During this phase, the effective potential is now given by

$V(|\phi|)=\left(m_{\phi}^{2}+\alpha R_{\mathrm{RH}}+\frac{64(1+3 w)}{27(1+w)^{4} \mathcal{M}^{2} t^{4}}\right)|\phi|^{2}+\lambda|\phi|^{4}$,

where $R_{\mathrm{RH}}$ denotes the Ricci scalar during reheating. During reheating, the Ricci scalar scales as

$$
R=\frac{4}{3}\left[\frac{1-3 w}{(1+w)^{2}}\right] t^{-2},
$$

for a constant equation of state, so that for $w<1 / 3$ the sign of this term is given by the sign of $\alpha$ (the same is true during inflation). Given that we end inflation in the symmetrybreaking minimum set by the Gauss-Bonnet coupling, there are three scenarios depending on the sign of $\alpha$. For $\alpha<0$, the Ricci coupling contributes a tachyonic mass for the scalar that redshifts at a slower rate than the tachyonic mass due to the Gauss-Bonnet coupling. This means that at some time $t_{\star} \sim \mathcal{M}^{-2}$ the symmetry-breaking effects are dominated by the Ricci coupling, and the evolution of the minimum will behave in a similar manner to that described by Ref. [21]. If, instead, the symmetry is restored before this term comes to dominate, then the Gauss-Bonnet term will dominate throughout. Since this scenario is novel, we will focus on this in what follows, although it would be interesting to study hybrid scenarios in the future. Finally, if $\alpha>0$, then the Ricci coupling is not responsible for symmetry breaking and, instead, adds a correction to the bare mass of the scalar that redshifts over time. Our analysis below will also encapsulate this scenario. Interestingly, in many string theory compactifications, scalars coupled to the dilaton arise without associated Ricci couplings [31,49]. For this reason, models commonly studied in the literature omit such couplings from the outset (e.g., [50-54]). Our analysis also applies to these models by construction.

We now proceed to calculate the baryon-to-photon ratio under the assumption that the Ricci coupling does not interfere with symmetry breaking. As discussed above, the main effect of this coupling is to correct the bare mass of the scalar. To parameterize our ignorance of this, we define

$$
\bar{m}_{\phi}^{2}=m_{\phi}^{2}+\alpha R_{\mathrm{RH}},
$$

which we take to be constant. We require $m_{\phi}<\mathcal{M}$ in order to be consistent with the effective field theory (allowing
$m_{\phi}>\mathcal{M}$ is tantamount to ignoring new light states that enter above the cutoff) but note that there is no such restriction on $\bar{m}_{\phi}$. With this in mind, the time-dependent minimum is given by

$$
\left|\phi_{\min }(t)\right|=\frac{\bar{m}_{\phi}}{\sqrt{2 \lambda}}\left[\left(\begin{array}{l}
\bar{t} \\
t
\end{array}\right)^{4}-1\right]^{1 / 2},
$$

where $\bar{t}$ is given by Eq. (9). The symmetry is restored at a time $t=\bar{t}$, and, for the sake of providing a concrete scenario, we assume that the equation of state crosses $w=-1 / 3$ at a time later than $\bar{t}$ so that the symmetry is restored due to the redshifting of the Gauss-Bonnet term but not due to the sign change that occurs when the equation of state exceeds $-1 / 3$. $^{3}$ The Hubble parameter when the symmetry is restored is

$$
\bar{H}=\frac{2}{3(1+w) \bar{t}} \sim \sqrt{\bar{m}_{\phi} \mathcal{M}} .
$$

In order to generate a net $B-L$, the angular field must begin to roll at around this time so that its mass $m_{\theta} \sim \bar{H}$. This gives the condition

$$
\bar{m}_{\theta}^{2}=\frac{\Delta V_{\theta \theta}}{|\phi|_{\min }^{2}} \sim \varepsilon_{0}|\phi|_{\min }^{2} \sim \bar{H}^{2},
$$

which allows us to fix the magnitude of the symmetrybreaking terms:

$$
\varepsilon_{0} \sim \frac{\bar{H}^{2}}{|\phi|_{\min }^{2}} \sim \lambda \frac{\mathcal{M}}{\bar{m}_{\phi}}
$$

The motion of the angular field generates the $B-L$ asymmetry. In particular, the time component of the conserved $\mathrm{U}(1)_{B-L}$ current, which corresponds to $n_{B-L}$, is

$$
n_{B-L}=j^{0}=2 q \operatorname{Im}\left(\phi^{\star} \partial^{0} \phi\right)=2 q|\phi|^{2} \dot{\theta},
$$

where $q$ is the $B-L$ charge of the scalar. One can see that angular motion is necessary to generate a net $B-L$. The equation of motion for the angular field can be expressed in the form

$$
\dot{n}_{B-L}+3 H n_{B-L}=2 q \varepsilon_{0}|\phi|^{4} \sin (4 \theta+\psi) .
$$

Approximating $\dot{n}_{B-L}$ by $H n_{B-L}$, using Eq. (26), and ignoring order-unity factors, we have

\footnotetext{
${ }^{3} \mathrm{~A}$ more stringent requirement is that the equation of state should exceed $-3 / 5$ at a time $t>\bar{t}$ so that the minimum is always a stable attractor. In practice, this distinction is negligible, since the field begins near the minimum and tracks it for a long time in both cases.
} 


$$
n_{B-L} \approx \varepsilon_{0} \frac{|\phi|^{4}}{\bar{H}} \sin (4 \theta+\psi) \sim|\phi|_{\min }^{2} \bar{H}
$$

Since baryogenesis occurs during reheating, the entropy density around this time is

$$
s \sim \frac{M_{\mathrm{pl}}{ }^{2} \bar{H}^{2}}{T_{\mathrm{RH}}}
$$

so that the ratio

$$
\begin{aligned}
\frac{n_{B-L}}{s} \sim & 10^{-10}\left(\frac{10^{-5}}{\lambda}\right)\left(\frac{T_{\mathrm{RH}}}{10^{12} \mathrm{GeV}}\right)\left(\frac{\bar{m}_{\phi}}{10^{8} \mathrm{GeV}}\right)^{3 / 2} \\
& \times\left(\frac{\mathcal{M}}{10^{6} \mathrm{GeV}}\right)^{-1 / 2} .
\end{aligned}
$$

Equation (30) is the main result of this section. It demonstrates that the mechanism that we have developed in this section can generate the observed matterantimatter asymmetry observed in the Universe with suitable parameter choices. As it stands, this asymmetry is entirely stored in the new scalar $\phi$, but, provided that the scalar can decay to the left-handed sector of the standard model, sphalerons will reprocess this into a net baryon and lepton asymmetry. The potential couplings of such scalars to the standard model were discussed at length in Ref. [24], and we have nothing new to add here. We simply remark that many such couplings are possible, and constraints from direct and indirect detection as well collider bounds are not yet at the level where such couplings are sufficiently forbidden.

Some comments are in order. First, we have made several approximations. This was both for simplicity (e.g., ignoring order-unity factors, assuming a constant equation of state $w$, etc.) and to make the calculation analytically tractable. For example, we approximated $\dot{n}_{B-L}$ by $H n_{B-L}$ and assumed that the evolution of the scale factor is given by the standard Friedmann equations without accounting for the backreaction of the Gauss-Bonnet term on the equation of motion of the metric. Said another way, we assumed that the contribution of the scalar field to the evolution of the Universe was subdominant throughout this entire process. This latter assumption is easy to verify. The contribution of the scalar potential to the Friedmann equation is

$$
\Omega_{\phi} \sim \frac{\bar{m}_{\phi}^{2}|\phi|^{2}}{M_{\mathrm{pl}}{ }^{2} H^{2}} \sim \frac{\bar{m}_{\phi}^{4}}{\lambda M_{\mathrm{pl}}{ }^{2} \bar{H}^{2}} \sim \frac{m_{\phi}^{3}}{\lambda M_{\mathrm{pl}}{ }^{2} \mathcal{M}} \lesssim 10^{-12},
$$

where we have used Eqs. (7) and (24) and the fiducial values needed to set every term in Eq. (30) to unity. Similarly, the contribution from the Gauss-Bonnet coupling is

$$
\Omega_{\mathcal{G}} \sim \frac{|\phi|^{2}}{\mathcal{M}^{2}} \frac{H^{4}+\dot{H} H^{2}}{H^{2} M_{\mathrm{pl}}{ }^{2}} \sim \frac{|\phi|^{2}}{M_{\mathrm{pl}}{ }^{2}} \frac{H^{2}}{\mathcal{M}^{2}} \lesssim 10^{-12} .
$$

Evidently, the backreaction is indeed negligible. The other assumptions require numerical computations to verify. We do precisely this in the next section, where we solve the equations of motion numerically and verify Eq. (30).

\section{Numerical examples}

In this section, we will verify numerically that baryogenesis during reheating from a scalar-Gauss-Bonnet coupling can indeed generate the net matter-antimatter asymmetry observed in the Universe as predicted analytically by Eq. (30), validating our approximations made in the previous sections. Since we have already established that the backreaction of the scalar on the Friedmann equations is negligible, we will solve only the equation of motion for the scalar

$$
\ddot{\phi}+3 H \dot{\phi}+\frac{\partial V_{\mathrm{eff}}\left(\phi, \phi^{\star}\right)}{\partial \phi^{\star}}=0,
$$

with $V_{\text {eff }}$ given by

$$
\begin{aligned}
V_{\text {eff }}\left(\phi, \phi^{\star}\right)= & \left(\bar{m}_{\phi}^{2}-24 \frac{H^{4}+H^{2} \dot{H}}{\mathcal{M}^{2}}\right)|\phi|^{2} \\
& +\lambda|\phi|^{4}+\frac{\varepsilon}{4} \phi^{4}+\frac{\varepsilon^{\star}}{4} \phi^{\star 4} ;
\end{aligned}
$$

i.e., we are now including the symmetry-breaking terms and the effective mass $\bar{m}$ in its definition. In order to be agnostic about the details of reheating, we parameterize the Hubble constant during reheating as

$$
H(t)=\frac{H_{I}}{\left[1+4\left(H_{I} t\right)^{2}\right]^{1 / 2}},
$$

so that it interpolates between a constant value $H_{I}$ during inflation and tends to $1 /(2 t)$ at late times, corresponding to a radiation-dominated universe $[55,56]$. The intermediate epoch is presumed to encapsulate reheating. Given the simple and smooth evolution, it is likely that this

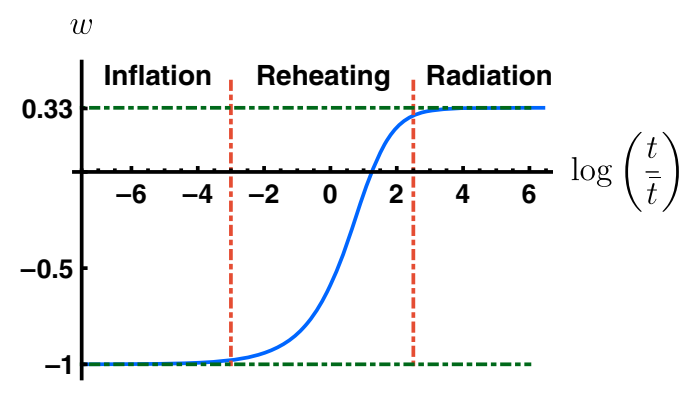

FIG. 1. The equation of state as a function of time that gives rise to the parameterization of the Hubble constant given in Eq. (35). 


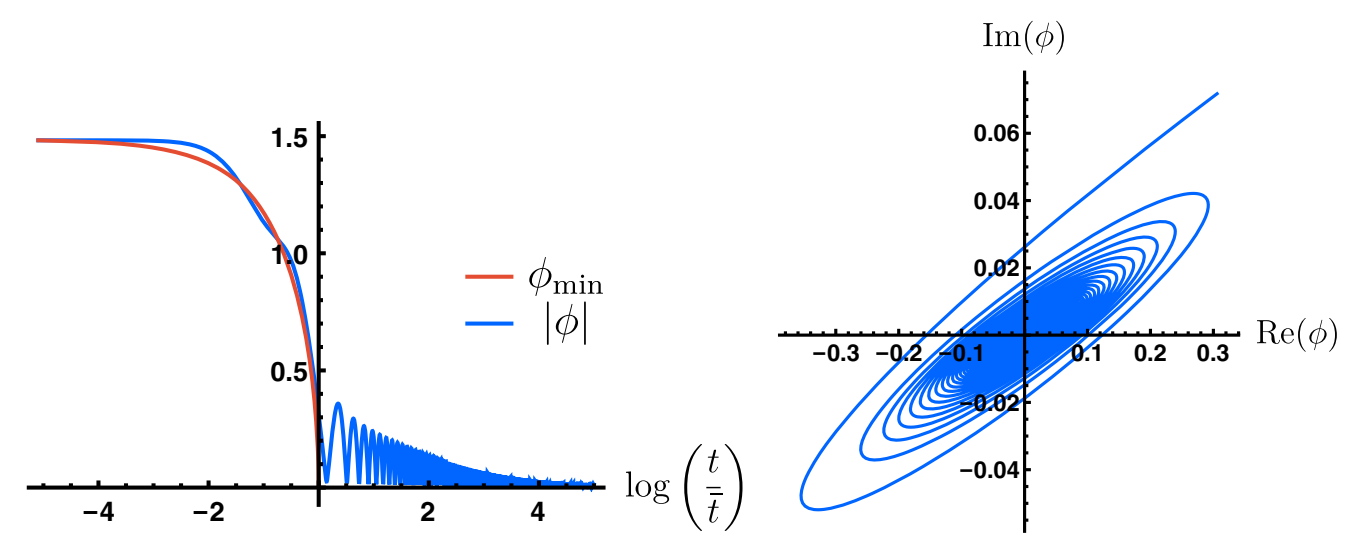

FIG. 2. Left: The evolution of $|\phi|_{\min }(t)$ calculated analytically [using the parameterization in Eq. (35)] and the results of the numerical integration for $|\phi|(t)$. Right: The evolution of real and imaginary parts of the scalar.

approximation corresponds to some form of perturbative reheating with the inflaton close to the minimum of its potential. One could use more complicated parameterizations to describe more complicated and nonperturbative scenarios. Note that there is no period where $H=2 / 3 t$ (i.e., $w=0$ ), so this parameterization does not include a potential with $V(\phi) \propto \phi^{2}$ near the minimum, which is the preferred scenario for Affleck-Dine baryogenesis. As discussed above, our mechanism would not work in this case, because the minimum would not be a stable attractor. The equation of state as a function of time for this parametrization is shown in Fig. 1. As a consistency check, we integrated the equations with several alternate parameterizations; all gave qualitatively similar results.

The results of our integrations are shown in Figs. 2 and 3. In all cases, we used the fiducial values $\bar{m}_{\phi}=$ $7 \times 10^{8} \mathrm{GeV}, \quad \mathcal{M}=10^{6} \mathrm{GeV}, \quad H_{I}=1.6 \times 10^{7} \mathrm{GeV}$, $T_{\mathrm{RH}}=2.1 \times 10^{12} \mathrm{GeV}, \lambda=3.3 \times 10^{-4}$, and $\varepsilon_{0}=3.2 \times 10^{-7}$ and checked that qualitatively similar results are obtained for other choices. The isocurvature bound given in Eq. (19) can also be satisfied for this parameter choice. The evolution of the scalar is shown in Fig. 2. The left panel shows the evolution of the scalar compared with the theoretical prediction for the evolution of the minimum. One can see that the field adiabatically tracks the minimum

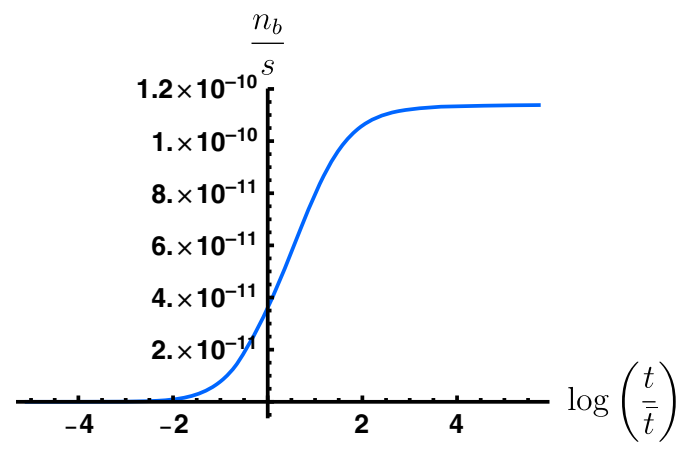

FIG. 3. The ratio $n_{B-L} / s$ as a function of time. until the symmetry is restored. The right panel shows the real and imaginary parts of the scalar, and the angular motion that generates the $B-L$ asymmetry is evident. Finally, we plot the ratio $n_{B-L} / s$ in Fig. 3. A value of the order of $10^{-10}$ is reached asymptotically, confirming our approximations in the previous sections and their ultimate analytical prediction given in Eq. (30). We have found that changing the initial conditions and the parameters yields qualitatively similar behavior.

\section{DISCUSSION AND CONCLUSIONS}

In this work, we have studied the effective field theory of a $U(1)$ scalar field coupled to both gravity and the inflaton. This theory contains several natural mechanisms for spontaneously breaking the U(1) symmetry, including the Affleck-Dine mechanism [4,17,18], the matter-induced breaking of Ref. [21], and a novel mechanism where a coupling to the Gauss-Bonnet invariant can induce a tachyonic instability for the scalar around the symmetrypreserving point in field space. Identifying the charge with $B-L$, we have then developed a novel scenario for baryogenesis that employs this breaking mechanism.

The Gauss-Bonnet term has some unique properties that give rise to several novel features compared with other scenarios that make use of cosmological dynamics to realize an inverse symmetry-breaking transition. First, the Gauss-Bonnet term has different signs depending on the equation of state, with the threshold given by $w=-1 / 3$. This means that the symmetry can be broken either during reheating and inflation or during the standard big bang cosmological history, but not both. Second, the contribution to the effective mass of the scalar redshifts like $t^{-4}$ rather than $t^{-2}$ as is the case for Affleck-Dine and matter-induced baryogenesis. We have shown that this causes the (time-dependent) minimum to be an attractor when $-1<w<-3 / 5$ and an unstable fixed point otherwise, signaling that any stable baryogenesis mechanism must happen during a cosmological epoch dominated by a 
component with an equation of state in this range, inflation and reheating being the patent epochs.

Using the above facts, we have constructed the following scenario: During inflation, the symmetry is broken and the radial part of the scalar minimizes its effective potential (the position of the minimum is constant), while the angular field is frozen due to Hubble damping. After reheating begins, the minimum of the effective potential begins to move towards zero as the Gauss-Bonnet term starts to redshift. At this time, the angular field begins to roll down its potential, which arises due to small $\mathrm{U}(1)_{B-L}$-breaking terms in the action, generating a net $B-L$. Finally, the symmetry is restored when the Gauss-Bonnet term has redshifted sufficiently, and the radial part of the field rapidly returns to zero. We have analytically predicted the amount of $B-L$ produced, demonstrating that the net matter-antimatter asymmetry observed in the Universe can be accounted for using reasonable parameter choices. We have also verified our analytic predictions using numerical computations.

Of course, this is just the first part of the story. In order to fully account for the observed asymmetry, the $B-L$ must be transferred into the left-handed sector of the standard model. This is not particularly difficult, and there are several portals to the standard model that are allowed by current constraints [21]. Any of these are sufficient for our purposes.

An important question is whether these theories can be tested observationally. Interestingly, theories of this kind exhibit a phenomenon known as spontaneous scalarization [50-54,57] (see also [58-61]), whereby black holes and neutron stars can suddenly acquire a large scalar charge (or, equivalently, hair) due to quadratic scalar-Gauss-Bonnet coupling. Indeed, it is precisely the same tachyonic instability we identified here that underlies this mechanism. So far, all of the studies of spontaneous scalarization have used real fields, so it would be interesting to generalize these to complex fields. In particular, Ref. [52] studied an identical EFT to Eq. (1) (without the inflaton coupling) with the exception that the scalar field there was real. The cutoff for theories that scalarize solar mass black holes is $\mathcal{M} \sim 10^{-22} \mathrm{GeV}(\sim 35 \mathrm{~km})$ [52], several orders of magnitude smaller than the cutoff for our baryogenesis mechanism. It would be interesting to explore which type of objects could become scalarized in our theory. Recently, Ref. [62] studied the most general subset of Horndeski theories that can give rise to a tachyonic mass for a real scalar field. It would be interesting to generalize this to complex scalars and determine whether any new baryogenesis mechanisms exist.

\section{ACKNOWLEDGMENTS}

J.S. is supported by the Center for Particle Cosmology at the University of Pennsylvania. The work of M. T. is supported in part by U.S. Department of Energy (HEP) Grant No. DE-SC0013528 and by NASA ATP Grant No. NNH17ZDA001N.
[1] M. Trodden, Rev. Mod. Phys. 71, 1463 (1999).

[2] A. Riotto and M. Trodden, Annu. Rev. Nucl. Part. Sci. 49, 35 (1999).

[3] J. M. Cline, in Les Houches Summer School-Session 86: Particle Physics and Cosmology: The Fabric of Spacetime Les Houches, France, 2006 (Elsevier, France, 2006).

[4] M. Dine and A. Kusenko, Rev. Mod. Phys. 76, 1 (2003).

[5] M. Trodden, eConf C040802, L018 (2004).

[6] D. E. Morrissey and M. J. Ramsey-Musolf, New J. Phys. 14, 125003 (2012).

[7] R. Allahverdi and A. Mazumdar, New J. Phys. 14, 125013 (2012).

[8] E. V. Arbuzova, Phys. Part. Nucl. Lett. 15, 348 (2018).

[9] J. Racker, Nucl. Part. Phys. Proc. 273-275, 334 (2016).

[10] Y. Cui, Mod. Phys. Lett. A 30, 1530028 (2015).

[11] B. D. Fields, P. Molaro, and S. Sarkar, Chin. Phys. C 38, 090001 (2014).

[12] R. H. Cyburt, B. D. Fields, K. A. Olive, and T.-H. Yeh, Rev. Mod. Phys. 88, 015004 (2016).

[13] G. Aad et al. (ATLAS and CMS Collaborations), Phys. Rev. Lett. 114, 191803 (2015).
[14] M.-C. Chen, in Proceedings of Theoretical Advanced Study Institute in Elementary Particle Physics: Exploring New Frontiers Using Colliders and Neutrinos (TASI 2006), Boulder, Colorado, 2006 (World Scientific, Hackensack, 2007), pp. 123-176.

[15] S. Davidson, E. Nardi, and Y. Nir, Phys. Rep. 466, 105 (2008).

[16] C. Balazs, arXiv:1411.3398.

[17] I. Affleck and M. Dine, Nucl. Phys. B249, 361 (1985).

[18] M. Dine, L. Randall, and S. D. Thomas, Nucl. Phys. B458, 291 (1996).

[19] R. Allahverdi, R. Brandenberger, F.-Y. Cyr-Racine, and A. Mazumdar, Annu. Rev. Nucl. Part. Sci. 60, 27 (2010).

[20] M. A. Amin, M. P. Hertzberg, D. I. Kaiser, and J. Karouby, Int. J. Mod. Phys. D 24, 1530003 (2015).

[21] J. Sakstein and M. Trodden, Phys. Lett. B 774, 183 (2017).

[22] D. Bettoni and J. Rubio, Phys. Lett. B 784, 122 (2018).

[23] M. Kurkov, Eur. Phys. J. C 76, 329 (2016).

[24] J. Sakstein and A. R. Solomon, Phys. Lett. B 773, 186 (2017).

[25] A. De Simone, T. Kobayashi, and S. Liberati, Phys. Rev. Lett. 118, 131101 (2017). 
[26] J. J. Heckman, C. Lawrie, L. Lin, J. Sakstein, and G. Zoccarato, arXiv:1901.10489.

[27] V. Faraoni, Phys. Rev. D 53, 6813 (1996).

[28] F. L. Bezrukov and M. Shaposhnikov, Phys. Lett. B 659, 703 (2008).

[29] F. Takahashi, arXiv:1505.07950.

[30] G. Alonso-lvarez and J. Jaeckel, J. Cosmol. Astropart. Phys. 10 (2018) 022.

[31] P. Kanti, N. E. Mavromatos, J. Rizos, K. Tamvakis, and E. Winstanley, Phys. Rev. D 54, 5049 (1996).

[32] S. Nojiri, S. D. Odintsov, and M. Sasaki, Phys. Rev. D 71, 123509 (2005).

[33] E. J. Copeland, M. Sami, and S. Tsujikawa, Int. J. Mod. Phys. D 15, 1753 (2006).

[34] S. Bahamonde, C. G. Bhmer, S. Carloni, E. J. Copeland, W. Fang, and N. Tamanini, Phys. Rep. 775-777, 1 (2018).

[35] Z. Yi, Y. Gong, and M. Sabir, Phys. Rev. D 98, 083521 (2018).

[36] S. Chakraborty, T. Paul, and S. SenGupta, Phys. Rev. D 98, 083539 (2018).

[37] S. D. Odintsov and V. K. Oikonomou, Phys. Rev. D 98, 044039 (2018).

[38] K. D. Lozanov, arXiv:1907.04402.

[39] T. P. Sotiriou and S.-Y. Zhou, Phys. Rev. Lett. 112, 251102 (2014).

[40] J. Sakstein and B. Jain, Phys. Rev. Lett. 119, 251303 (2017).

[41] T. Baker, E. Bellini, P. G. Ferreira, M. Lagos, J. Noller, and I. Sawicki, Phys. Rev. Lett. 119, 251301 (2017).

[42] P. Creminelli and F. Vernizzi, Phys. Rev. Lett. 119, 251302 (2017).

[43] J. M. Ezquiaga and M. Zumalacárregui, Phys. Rev. Lett. 119, 251304 (2017).

[44] N. Franchini and T. P. Sotiriou, arXiv:1903.05427.

[45] Y. Akrami et al. (Planck Collaboration), arXiv:1807.06211
[46] K. Enqvist and J. McDonald, Phys. Rev. Lett. 83, 2510 (1999).

[47] K. Enqvist and J. McDonald, Phys. Rev. D 62, 043502 (2000).

[48] M. Kawasaki and F. Takahashi, Phys. Lett. B 516, 388 (2001).

[49] R. R. Metsaev and A. A. Tseytlin, Nucl. Phys. B293, 385 (1987).

[50] H. O. Silva, J. Sakstein, L. Gualtieri, T. P. Sotiriou, and E. Berti, Phys. Rev. Lett. 120, 131104 (2018).

[51] H. O. Silva, C. F. B. Macedo, T. P. Sotiriou, L. Gualtieri, J. Sakstein, and E. Berti, Phys. Rev. D 99, 064011 (2019).

[52] C. F. B. Macedo, J. Sakstein, E. Berti, L. Gualtieri, H. O. Silva, and T. P. Sotiriou, Phys. Rev. D 99, 104041 (2019).

[53] D. D. Doneva and S. S. Yazadjiev, Phys. Rev. Lett. 120, 131103 (2018).

[54] D. D. Doneva and S. S. Yazadjiev, J. Cosmol. Astropart. Phys. 04 (2018) 011.

[55] D. I. Podolsky, G. N. Felder, L. Kofman, and M. Peloso, Phys. Rev. D 73, 023501 (2006).

[56] K. D. Lozanov and M. A. Amin, Phys. Rev. Lett. 119, 061301 (2017).

[57] M. Minamitsuji and T. Ikeda, Phys. Rev. D 99, 044017 (2019).

[58] G. Antoniou, A. Bakopoulos, and P. Kanti, Phys. Rev. Lett. 120, 131102 (2018).

[59] G. Antoniou, A. Bakopoulos, and P. Kanti, Phys. Rev. D 97, 084037 (2018).

[60] A. Bakopoulos, G. Antoniou, and P. Kanti, Phys. Rev. D 99, 064003 (2019).

[61] T. Anson, E. Babichev, C. Charmousis, and S. Ramazanov, J. Cosmol. Astropart. Phys. 06 (2019) 023.

[62] N. Andreou, N. Franchini, G. Ventagli, and T. P. Sotiriou, Phys. Rev. D 99, 124022 (2019). 